\title{
Vitrification of camel skin tissue for use as a resource for somatic cell nuclear transfer in Camelus dromedarius
}

\author{
Young-Bum Son ${ }^{1} \cdot$ Yeon Ik Jeong ${ }^{1} \cdot$ Yeon Woo Jeong ${ }^{1} \cdot$ Xianfeng Yu $^{2} \cdot$ Lian Cai $^{1} \cdot$ Eun Ji Choi ${ }^{1}$. \\ Mohammad Shamim Hossein ${ }^{1} \cdot$ Alex Tinson $^{3} \cdot$ Kuhad Kuldip Singh $^{3} \cdot$ Singh Rajesh $^{3} \cdot$ Al Shamsi Noura $^{3}$. \\ Woo Suk Hwang ${ }^{1}$ (it)
}

Received: 25 March 2021 / Accepted: 2 May 2021 / Published online: 20 May 2021 / Editor: Tetsuji Okamoto

(C) The Author(s) 2021

Animals with superior genetics could be reproduced by somatic cell nuclear transfer (SCNT). Generally, for SCNT viable cell lines are established from living animals (Jeong et al. 2020). However, complication arises when animals die suddenly due to a disease or accident (Jeong et al. 2013). A suitable tissue cryopreservation method that can preserve tissue integrity is important. The cryopreservation is a prominent method for preserving animal genetic resources, and autologous cells could be obtained from cryopreserved tissue which has numerous clinical applications (Buck et al. 1981; Taylor et al. 2019). Therefore, studies on tissue cryopreservation in several species, such as humans, rats, sheep, and dog, have been reported (Ishijima et al. 2006; Wang et al. 2008; Courbiere et al. 2009; Deng et al. 2009). However, studies have not been reported on the establishment of cells from cryopreserved tissues of Camelus dromedarius (camel). In addition, limited studies have been reported on cloning through the SCNT technique using cells established from cryopreserved tissues without cryoprotectants (Hoshino et al. 2009; Zhang et al. 2012; Jeong et al. 2020).

Tissue cryopreservation is largely a slow-freezing and vitrification method. In general, programmed slow freezing is used for tissue freezing (Santos et al. 2007; Dalman et al. 2017; Lee et al. 2019). However, this technique requires long time for cryopreservation which could be detrimental (Santos

Woo Suk Hwang

hwangws@adbrf.org

1 UAE Biotech Research Center, 30310 Al Wathba, Abu Dhabi, United Arab Emirates

2 Jilin Provincial Key Laboratory of Animal Model, College of Animal Science, Jilin University, Changchun, China

3 Hilli E.T. Cloning and Surgical Centre Presidential Camels and Camel Racing Affairs, 17292 Al-Ain, United Arab Emirates et al. 2007; Wang et al. 2008; Dalman et al. 2017; Lee et al. 2019). Vitrification is a simple, inexpensive and rapid method for cryopreservation (Dalman et al. 2017; Lee et al. 2019; Santos et al. 2007; Wang et al. 2008). It could replace the slow freezing techniques as it prevents the formation of ice crystals due to an ultrarapid cooling procedure (Amorim et al. 2011). For vitrification of tissues, intracellular cryoprotectants such as dimethyl-sulfoxide (Me2SO), ethylene glycol (EG), and glycerol are mainly used (Santos et al. 2007; Amorim et al. 2011). Among these, EG has the lowest cytotoxicity and a rapid permeability across the cell membrane (Bautista and Kanagawa 1998; Eto et al. 2014; Kuleshova et al. 1999). Furthermore, EG leads to thinning of the phospholipid biolayers and diffuses through them (Hughes and Mancera 2014). Therefore, tissue vitrification using EG has been reported in various mammals (Isachenko et al. 2003; Yeoman et al. 2005; Gandolfi et al. 2006). However, studies on cryopreservation of tissue, except for ovarian tissue, are very limited, and no studies on tissue vitrification using EG in camels have been reported. Therefore, the present study established cell lines from vitrified camel skin tissues, their characteristics were evaluated accordingly, and the developmental efficiency of the embryo was analyzed after performing SCNT using these cells as donor cells.

All animal work was performed according to the animal study guidelines which were approved by the ethics committee at the Management of Scientific Centers and Presidential Camels (Accession No: PC4.1.5). To preserve the genetic resources of a camel (male) that died suddenly, vitrification was performed after biopsy of the ear skin tissue. As a control for evaluating the effectiveness of the tissue vitrification method, ear skin tissues were collected from 6 different camels (three males and three females). The chemicals used in this study were purchased from Sigma (St. Louis, MO) unless otherwise noted. We adjusted the $\mathrm{pH}$ to 7.4 and the osmolality to $280 \mathrm{mOsm} / \mathrm{kg}$ for all media. 
We performed tissue vitrification as previously described with minor modifications (Santos et al. 2007). Briefly, tissues were washed with DPBS (Life Technologies, Carlsbad, CA) containing $10 \mu \mathrm{g} / \mathrm{mL}$ penicillin/streptomycin solution (Invitrogen). After that, tissues were cut into small pieces (1 $\mathrm{cm}^{3}$ ) at $25^{\circ} \mathrm{C}$. The fragments were exposed to Dulbecco's modified Eagle's medium (DMEM; Thermo Fisher scientific, Waltham, MA) supplemented with $20 \%$ EG and $10 \%$ fetal bovine serum (FBS; Invitrogen) for $1 \mathrm{~min}$ and transferred to DMEM supplemented with 40\% EG and 10\% FBS using cryo-vials (Nunc, Roskilde, Denmark). The tissues were immediately transferred to liquid nitrogen $\left(-196^{\circ} \mathrm{C}\right)$ and stored from 2010 to 2019. Both fresh and vitrified camel ear skin tissues were fixed with $10 \%$ formalin and embedded in paraffin. For histologic assessment, they were stained with hematoxylin and eosin (H\&E) and the structure of ear skin tissues was identified as being in a normal state in both tissues (Fig. $1 a)$.

The thawing of tissues was also conducted as previously reported with minor modifications (Santos et al. 2007). In brief, to remove the cryoprotectant during the thawing process, the vitrified tissues were kept at room temperature for $5 \mathrm{~min}$ and transferred to DMEM supplemented with $0.3 \mathrm{M}$ sucrose and $10 \% \mathrm{FBS}$ at $38^{\circ} \mathrm{C}$ for $5 \mathrm{~min}$ followed by DMEM supplemented with $0.15 \mathrm{M}$ sucrose and $10 \% \mathrm{FBS}$ for $5 \mathrm{~min}$. After that, the tissues were washed 3 times using DMEM containing $10 \%$ FBS. We minced and treated with enzymatic dissociation of the vitrified and fresh tissues. After that, the cells were subsequently cultured in DMEM containing $10 \%$ FBS, $1 \%$ nonessential amino acids (Thermo Fisher Scientific, Waltham, MA), $1 \%$ antibiotic-antimycotic, and $0.1 \% \beta$ mercaptoethanol (Thermo Fisher Scientific) at $38^{\circ} \mathrm{C}$ in a humidified atmosphere of $5 \% \mathrm{CO}_{2}$. The number of viable cells and the cell proliferation capacity from vitrified and fresh tissues were analyzed as previously described with minor modifications (Legzdina et al. 2016; Shivakumar et al. 2016). Fibroblasts derived from both tissues were stained with $0.4 \%$ Trypan blue and the number of cells was counted every $2 \mathrm{~d}$. Fibroblasts $\left(2 \times 10^{3}\right)$ from both tissues were seeded into 6well plates, and after every $72 \mathrm{~h}$, the cells were counted using a hemocytometer. The population of doubling time (PDT) of the cells was calculated in accordance with the formula, $\mathrm{PDT}=\log 2 \times \mathrm{T} /(\log \mathrm{NH}-\log \mathrm{NI})$, where $\mathrm{T}$ is the culture time, $\log \mathrm{NH}$ is the harvest cell number, and $\log \mathrm{NI}$ is the initial cell number. Camel fibroblasts from fresh and vitrified skin tissues showed homogenous plate-adherent and spindle-like cell morphology (Fig. 1b,c). In the initial culture process, fibroblasts derived from fresh tissues showed enhanced growth patterns compared with those derived from vitrified tissues (Fig. 1d). However, cell proliferation assessed by PDT showed similar values of proliferation after passage 1 (Fig. 1e). Therefore, we assumed that fibroblasts isolated from vitrified tissues could be used as donor cells in the SCNT process, and analyzed the characteristics of the cells.

Cells lose their viability due to apoptosis induced during the tissue cryopreservation and thawing process (Amorim et al. 2011). This was one of the limitations of using tissue cryopreservation for securing adequate viable cells. Therefore, we cultured fibroblasts up to passage 3 for homogenization to use as donor cells for SCNT and analyzed cellular apoptosis that could have been induced by cryopreservation and the thawing of tissues. When cells from both fresh and vitrified tissues reached $80 \%$ confluence, senescence $\beta$-galactosidase (SA- $\beta$ gal) was analyzed using the senescence $\beta$-galactosidase
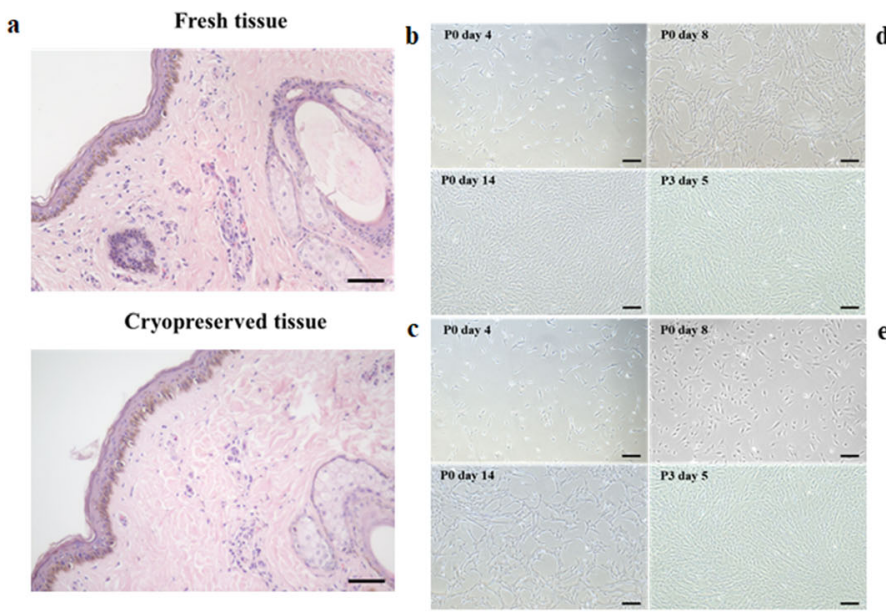

Figure 1. (a) H\&E staining was conducted. Both tissues showed normal H\&E staining. Scale bar $=800 \mu \mathrm{m}$. Morphology of camel fibroblasts from $(b)$ fresh and $(c)$ vitrified ear skin tissues (cryo), $(d)$ cell proliferation analysis, and (e) population of doubling time assay (PDT). The fibroblasts from both groups showed a similar spindle-like morphology. The proliferative ability was decreased in the cryo group compared to the fresh group at the initiation of culture and at passage 1. However,

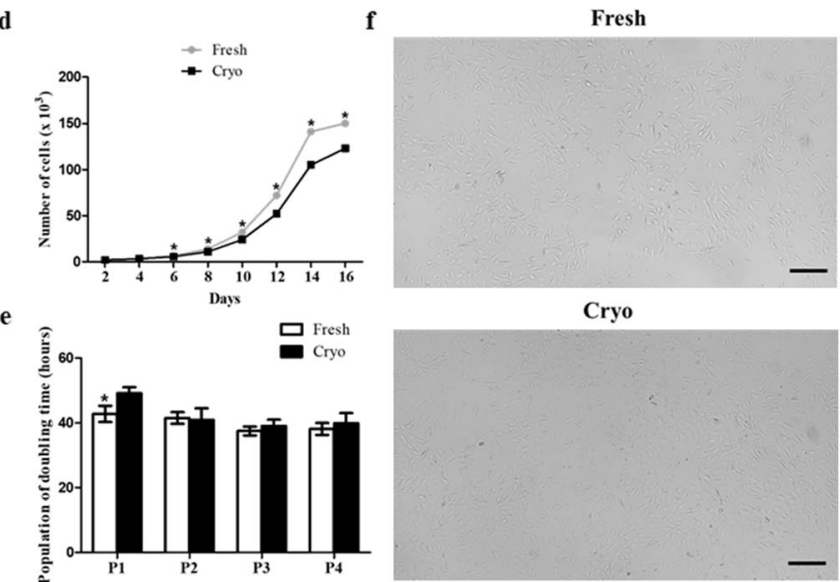

there was no significant difference in the proliferative capacity between the two groups after passage 1. Scale bar $=200 \mu \mathrm{m}$. Data are represented by the mean $\pm \mathrm{SD}$ of four independent experiments. Asterisks $(*)$ indicate significant differences between groups $(P<0.05)$. $(f)$ Cellular senescence was analyzed by SA- $\beta$ gal staining. The number of SA- $\beta$ gal positive cells was not different between the fresh- and the cryo groups. Scale bar $=200 \mu \mathrm{m}$. 
staining kit (Cell Signaling, Danvers, MA) following the manufacture's protocol. The proportion of SA- $\beta$ gal positive cells, an indicator of cellular senescence, was not different between the two groups (Fig. 1f) (Morgunova et al. 2016). To evaluate the conditions of the cells, we conducted cell cycle analysis following previous reports (Yang et al. 2006; Son et al. 2021). In brief, cells were fixed with $70 \%$ ethanol at $4{ }^{\circ} \mathrm{C}$ overnight. After that, the cell pellet was washed with DPBS and treated with $100 \mu \mathrm{g} / \mathrm{ml}$ RNase A to ensure that only DNA was stained. Finally, we stained detached cells $\left(1 \times 10^{6}\right)$ with propidium iodide solution $(10 \mu \mathrm{g} / \mathrm{ml})$, immediately analyzed them using flow cytometry (BD FACSVerseTM, BD Biosciences, Franklin Lakes, NJ), and categorized them into $\mathrm{G} 0 / \mathrm{G} 1, \mathrm{~S}$, and G2/M phases. The rate of cell apoptosis was also evaluated using a Dead Cell FITC Annexin V Apoptosis Detection Kit (Invitrogen) according to the manufacturer's instructions. The proportion of cells in the G0/G1 phase, which indicates cellular arrest, and $\mathrm{S}$ phase, the period in which cellular DNA is replicated, was not significantly different between the groups (Fig. 2a). Our results also showed that there was no significant difference in the portion of viable, early and late apoptosis between the two groups (Fig. 2b). Expression of apoptotic (BAX and p53), and anti-apoptotic (BAK) genes in fibroblasts derived from vitrified and fresh tissues was assessed using a real-time quantitative polymerase chain reaction (RT-qPCR). Total RNA was extracted from both cell lines at passage 3 from fresh and vitrified skin tissues using an easy-spin Total RNA Extraction Kit (Intron, Seongnam, Korea), and the synthesis of complementary DNA (cDNA) from total purified RNA $(2 \mu \mathrm{g})$ with $10 \mu \mathrm{M}$ oilgo(dT) primer at $42^{\circ} \mathrm{C}$ for $1 \mathrm{~h}$ using HisenScript RT PreMix kit (Intron). We performed qRT-PCR using a Rotor-Gene Q cycler (Qiagen, Hilden, Germany) and RealMOD ${ }^{\mathrm{TM}}$ Green AP 5x qPCR mix (Intron) containing 100 ng cDNA and specific primer sets (Table 1). The RT-qPCR setting included initial activation at $95^{\circ} \mathrm{C}$ for $12 \mathrm{~min}$, followed by $95^{\circ} \mathrm{C}$ for $15 \mathrm{~s}, 60^{\circ} \mathrm{C}$ for $25 \mathrm{~s}$, and $72^{\circ} \mathrm{C}$ for $25 \mathrm{~s}$. The expression of the gene was normalized to the mRNA levels of a control gene, glyceraldehyde 3-phosphate dehydrogenase (GAPDH). Pro-apoptotic-related (Bax, and p53) and antiapoptotic-related (BAK) genes, assessed by RT-qPCR, were also not significantly different in these groups (Fig. $2 c$ ). These results showed that the vitrification of tissue method performed in this study did not significantly influence the expression level of pro-apoptotic and anti-apoptotic factors.

During the freezing and thawing process, cells are exposed to various physical and chemical stresses and damaged by reactive oxygen species (ROS) (Len et al. 2019). These ROS affect the cellular membrane and mitochondria and influence ATP synthesis through oxidative phosphorylation (OXPHOS) in the mitochondria of eukaryotes (Len et al. 2019). Therefore, we analyzed the mitochondrial membrane potential and oxygen consumption rate (OCR), an indicator of mitochondrial respiration in fibroblasts. We stained cells with $\mathrm{JC}-1$ to evaluate the difference in mitochondrial membrane potential of the fibroblasts derived from fresh and vitrified tissues using a JC-1 mitochondrial membrane potential assay kit (Abnova, Taipei, Taiwan). After that, we analyzed the values of red-fluorescence aggregation, which indicates increased mitochondrial membrane potential, and greenfluorescence monomers, indicating decreased mitochondrial membrane potential (Fig. 2d). The ratio of red/green fluorescence histograms showed no significant difference between these groups (Fig. 2e). To confirm cellular respiration and energetics in fibroblasts from fresh and vitrified tissues, we conducted oxygen consumption rate analysis using a Seahorse XFp Cell Mito Stress Test (Seahorse, Agilent Technologies, Santa Clara, CA) following the manufacture's protocol. Both types of fibroblasts were seeded at 50,000 cells/well in a Seahorse XF24 plate and cultured for $24 \mathrm{~h}$ before the assay. XF24 media supplemented with $2 \mu \mathrm{M}$ rotenone, $1 \mu \mathrm{M}$ carbonyl cyanide-4 (trifluoromethoxy) phenylhydrazone (FCCP), and $1 \mu \mathrm{g} / \mathrm{ml}$ oligomycin was loaded into the accompanying cartridge. Treatment with the drugs in the medium occurred at the time points specified (Fig. $2 f$ ). The OCR was monitored using a Seahorse Bioscience XF24 Extracellular Flux Analyzer. Each cycle was performed as follows: mix for $3 \mathrm{~min}$, delay for $2 \mathrm{~min}$, and then measure for $3 \mathrm{~min}$. Basal respiration was evaluated before oligomycin injection, and proton leakage was calculated after oligomycin injection. ATP production was measured through the difference between basal respiration and proton leakage, and after FCCP injection, spare respiratory capacity was calculated through the difference between maximal respiration and ATP production. The OCR value was dramatically decreased after inhibition of the F0 ATP complex by treatment with oligomycin. After that, the OCR value was increased after the addition of FCCP, indicating that mitochondrial respiration was uncoupled in both groups. Our results revealed that the basal OCR indicating oxidative phosphorylation (OXPHOS) was not different in these groups (Fig. 2f). Furthermore, the values of basal respiration, spare respiratory capacity, proton leakage, and ATP production were also not significantly different between the groups (Fig. 2g). Overall, our data demonstrated that cells from vitrified tissues showed normal mitochondrial function compared to cells from fresh tissues.

We used fibroblasts derived from fresh and vitrified tissues as donor cells for SCNT. A total of 580 in vitro matured oocytes (96 ovaries) and 978 in vivo matured oocytes (78 oocyte donors) aged between 4 and 7 yr weighting 400 to $450 \mathrm{~kg}$ were used to evaluate the effect of donor cells on embryonic development after SCNT. The donors were injected with 5000 IU PMSG (Ceva, Libourne, France) and $500 \mu \mathrm{g}$ of closprostenol (Jurox, Rutherford, Australia) to stimulate the ovary as previously reported (Tinson et al. 2000). After that, the donors were injected with $100 \mu \mathrm{g}$ gonadorelin 
Table 1. List of primers used in the RT-qPCR analysis

\begin{tabular}{lllc}
\hline Gene name (symbol) & Primers sequence & Product size (bp) & Anneal. Temp $\left({ }^{\circ} \mathrm{C}\right)$ \\
\hline BCL2-associated X protein (BAX) & F: CTGAGCAGATCATGAAGAC & 171 & 60 \\
& R: TACTGTCGAGTTCATCTCC & 169 & 60 \\
BCL-2 antagonist/killer 1 (BAK) & F: TACGACTCAGAGTTCCAG & & \\
& R: GCTGGTAGACATGTAGGG & 222 & 60 \\
Tumor protein 53 (p53) & F: CCATCTACAAGAAGTCAGAG & \\
Glyceraldehyde 3-phosphate dehydrogenase (GAPDH) & R: AGTGGATAGTGGTACAGTCA & 133 \\
& F: GCTGAGTACGTTGTGGAGTC & 60 \\
\hline
\end{tabular}

acetate (Vetoquinol, Paris, France) 25 to $28 \mathrm{~h}$ before OPU was conducted. The oocytes were obtained by an Aloka Ultrasound Unit (Aloka) with a needle guide (Aloka, Tokyo, Japan) using 60-cm, 18-gauge lumen needle in the follicles. Ovaries were washed with $0.9 \%$ saline solution, and follicles were aspirated using an 18-gauge needle with a 10 -ml disposable syringe. The homogenous cytoplasm enclosed by at least three layers of compact cumulus cells was collected and washed with DPBS containing $5 \mathrm{mg} / \mathrm{ml}$ bovine serum albumin (BSA; Thermo Fisher Scientific) and 1\% antibioticantimycotic. For in vitro maturation (IVM), COCs were cultured at $38^{\circ} \mathrm{C}$ in $5 \% \mathrm{CO}_{2}$ in a humidified incubator for $42 \mathrm{~h}$ with IVM medium (IVF Bioscience, Falmouth, UK). After that, we conducted SCNT by the techniques as previously reported with minor modifications using in vitro and in vivo matured oocytes (Kim et al. 2012). The cumulus cells were denuded from oocytes by gentle pipetting with $0.1 \%$ hyaluronidase. The denuded oocytes were stained with $5 \mu \mathrm{g} / \mathrm{ml}$ bisbenzimide for $3 \mathrm{~min}$ and enucleated by aspirating the first polar body. After enucleation, a single donor cell was microinjected into the perivitelline space of the oocytes. These oocyte couplets were fused in fusion media containing $0.26 \mathrm{M}$ mannitol, $0.1 \mathrm{mM} \mathrm{MgSO}{ }_{4}, 0.5 \mathrm{mM}$ HEPES, and $0.05 \%(\mathrm{w} / \mathrm{v})$ BSA with two DC pulses of $1.8 \mathrm{kV} / \mathrm{cm}$ for 15 $\mu$ sec using a BTX Electro Cell Manipulator (BTX Inc., San Diego, CA). The reconstructed embryos were treated with $5 \mu \mathrm{M}$ ionomycin for $3 \mathrm{~min}$ and with $2.0 \mathrm{mM} 6$ dimethylaminopurine (6-DMAP) in BO-IVC (IVF Bioscience, Falmouth, UK) in a humidified incubator with $5 \% \mathrm{CO}_{2}$ at $39^{\circ} \mathrm{C}$ for $4 \mathrm{~h}$. After activation, we cultured the embryos in 6 to 8 oil-covered droplets at $38^{\circ} \mathrm{C}$ in a humidified incubator with $5 \% \mathrm{CO}_{2}$ and $5 \% \mathrm{O}_{2}$ for $7 \mathrm{~d}$. Our results showed that the rates of fused oocytes and cleaved embryos were similar in the fresh and cryopreserved groups (Table 2). The efficiency of blastocyst formation was slightly increased in the fresh group compared to the cryopreserved groups, but there
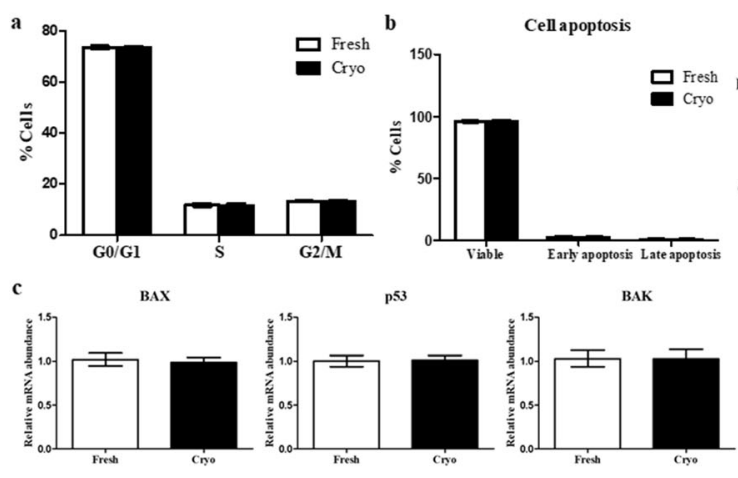

Figure 2. (a), (b) Analysis of the cell cycle and apoptosis in fibroblasts from fresh and vitrified ear skin tissues (cryo) at passage 3 . No differences were observed between the fresh and cryo groups with respect to the portion of the cell cycle $(a)$ and cellular apoptosis $(b)$. (c) The mRNA expression levels of apoptosis-specific genes (Bax, Bak, and p53) were similar in both the fresh and cryo groups. Data are presented as the mean \pm SD of four independent experiments. $(d),(e)$ Analysis of mitochondrial membrane potential $(\Delta \Psi \mathrm{m})$ in fibroblasts from fresh and vitrified ear skin tissues (cryo). (d) Both types of fibroblasts were stained with $\mathrm{JC}-1$
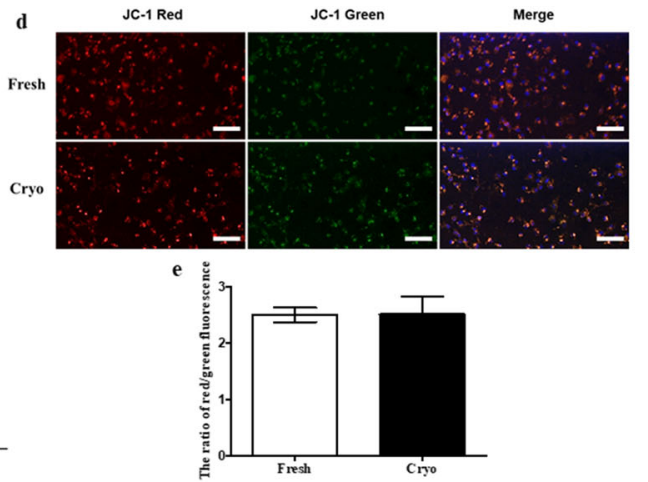

fluorescent dyes and DAPI. Scale bar $=100 \mu \mathrm{m}$. (e) The portion of $\mathrm{red} /$ green optical density was not significantly different between the fresh and cryo groups. Data are presented as the mean $\pm \mathrm{SD}$ of four independent experiments. $(f),(g)$ Oxygen consumption rate (OCR) assay of fibroblasts from fresh and vitrified ear skin tissues (cryo). $(f)$ The cryo group showed similar mitochondrial respiration compared to the fresh group. $(g)$ The values for basal respiration, proton leakage, spare respiratory capacity, and ATP production were similar in both groups. The data point in OCR represents the mean $\pm \mathrm{SD} ; n=10$ wells in independent experiments. 
Table 2. Effect of donor cells on the fusion rate and efficiency of embryo development of somatic cell nuclear transfer using in vitro and in vivo matured oocytes

Nuclear transfer

\begin{tabular}{llllll}
\hline Donor cells & Source of oocytes & No. of oocyte & & & \\
& & Reconstructed oocytes & Fused (\%) & Cleaved (\%) & Blastocyst (\%) \\
Fresh & In vitro matured oocytes & 301 & $213(72.13 \pm 3.86)$ & $136(65.83 \pm 5.60)^{\mathrm{a}}$ & $43(22.55 \pm 2.83)^{\mathrm{a}}$ \\
Cryo & & 279 & $196(71.38 \pm 1.86)$ & $122(62.47 \pm 2.89)^{\mathrm{a}}$ & $41(20.69 \pm 1.16)^{\mathrm{a}}$ \\
Fresh & In vivo matured oocytes & 507 & $385(75.85 \pm 1.32)$ & $285(76.93 \pm 1.98)^{\mathrm{b}}$ & $191(45.89 \pm 1.85)^{\mathrm{b}}$ \\
Cryo & & 471 & $348(75.79 \pm 2.26)$ & $292(74.91 \pm 3.13)^{\mathrm{b}}$ & $183(44.97 \pm 3.05)^{\mathrm{b}}$ \\
\hline
\end{tabular}

Data are represented by the mean $\pm \mathrm{SE}$ of four independent experiments

Lettered subscripts indicate statistical differences between groups $(P<0.05)$

was no significant difference (Table 2). The efficiency of fusion, cleavage, and blastocyst formation showed similar patterns in both the in vitro and in vivo matured oocytes (Table 2). Furthermore, similar to previous studies, the in vivo matured oocyte group showed higher cleavage and blastocyst formation rates than the in vitro matured oocyte group (Wani et al. 2018). Therefore, we confirmed that the source of oocytes was not related to the effects of the donor cells.

In conclusion, fibroblasts from vitrified tissues showed decreased proliferation compared with cells from fresh tissues in the initial culture process but showed similar patterns after passaging. Furthermore, our data showed similar values of mitochondrial metabolism, and the expression patterns of the cell cycle and apoptosis were also similarly observed after homogenization. Therefore, we determined that cells from vitrified tissues were suitable donor cells for SCNT, and confirmed that the efficiency of embryo development was similar to that of the fresh group. Taken together, camel SCNT embryos using cells from vitrified tissues were successfully developed to the blastocyst stage. These results suggested new approaches to tissue vitrification in preserving genetic resources.

Acknowledgements This project was supported by the Patronage of H.H. Sheikh Mansour bin Zayed Al Nahyan, Deputy Prime Minister of the U.A.E. and the Minister of Presidential Affairs, and we acknowledge his support and inspiration in the initiation and mentoring of this project, without whom this project would not have been possible.

Open Access This article is licensed under a Creative Commons Attribution 4.0 International License, which permits use, sharing, adaptation, distribution and reproduction in any medium or format, as long as you give appropriate credit to the original author(s) and the source, provide a link to the Creative Commons licence, and indicate if changes were made. The images or other third party material in this article are included in the article's Creative Commons licence, unless indicated otherwise in a credit line to the material. If material is not included in the article's Creative Commons licence and your intended use is not permitted by statutory regulation or exceeds the permitted use, you will need to obtain permission directly from the copyright holder. To view a copy of this licence, visit http://creativecommons.org/licenses/by/4.0/.

\section{References}

Amorim CA, Curaba M, Langendonckt AV, Dolmans MM, Donnez J (2011) Vitrification as an alternative means of cryopreserving ovarian tissue. Reprod Biomed Online 23:160-186

Bautista JA, Kanagawa H (1998) Current status of vitrification of embryos and oocytes in domestic animals: ethylene glycol as an emerging cryoprotectant of choice. Jpn J Vet Res 45:183-191

Buck DW, Hanssens LK, Kennett RH, Mellman WJ (1981) Successful cryopreservation of tissue and skin fibroblast cultures from patients with mucolipidosis II (I-cell disease). Cytogenet Cell Genet 29:9-15

Courbiere B, Caquant L, Mazoyer C, Franck M, Lornage J, Salle B (2009) Difficulties improving ovarian functional recovery by microvascular transplantation and whole ovary vitrification. Fertil Steril 91:2697-2706

Dalman A, Farahani NSDG, Totonchi M, Pirjani R, Ebrahimi B, Valojerdi MR (2017) Slow freezing versus vitrification technique for human ovarian tissue cryopreservation: An evaluation of histological changes, WNT signaling pathway and apoptotic genes expression. Cryobiology 79:29-36

Deng X, Zheng H, Yu X, Yu H, Zhang C, Chao L, Li R, Liu W (2009) Cryopreserved ovarian tissues can maintain a long-term function after heterotopic autotransplantation in rat. Reproduction 138:519 525

Eto T, Takahashi R, Kamisako T, Hioki K, Sotomaru Y (2014) A study on cryoprotectant solution suitable for vitrification of rat two-cell stage embryos. Cryobiology 68:147-151

Gandolfi F, Paffoni A, Brambilla EP, Bonetti S, Brevini TAL, Ragni G (2006) Efficiency of equilibrium cooling and vitrification procedures for the cryopreservation of ovarian tissue: comparative analysis between human and animal models. Fertil Steril 85:1150-1156

Hoshino Y, Hayashi N, Taniguchi S, Kobayashi N, Sakai K, Otani T, Iritani A, Saeki K (2009) Resurrection of a bull by cloning from organs frozen without cryoprotectant in a $-80^{\circ} \mathrm{C}$ freezer for a decade. Plos One 4:e4142

Hughes ZE, Mancera RL (2014) Molecular mechanism of the synergistic effects of vitrification solutions on the stability of phospholipid bilayers. Biophys J 106:2617-2624

Isachenko E, Isachenko V, Rahimi G, Nawroth F (2003) Cryopreservation of human ovarian tissue by direct plunging into liquid nitrogen. Eur J Obstet Gynecol Reprod Biol 108:186-193

Ishijima T, Kobayashi Y, Lee DS, Ueta YY, Matsui M, Lee JY, Suwa Y, Miyahara K, Suzuki H (2006) Cryopreservation of canine ovaries by vitrification. J Reprod Dev 52:293-299

Jeong YH, Park CH, Jang GH, Jeong YI, Hwang IS, Jeong YW, Kim YK, Shin T, Kim NH, Hyun SH, Jeung EB, Hwang WS (2013) Production of multiple transgenic Yucatan miniature pigs 
expressing human complement regulatory factors, human CD55, CD59, and H-transferase genes. Plos One 8:e63241

Jeong YI, Olson PO, Lian C, Lee ES, Jeong YW, Hwang WS (2020) Dog cloning from post-mortem tissue frozen without cryoprotectant. Cryobiology 97:226-230

Kim HS, Lee JY, Jeong EJ, Yang CJ, Hyun SH, Shin T, Hwang WS (2012) Effects of repetitive ionomycin treatment on in vitro development of bovine somatic cell nuclear transfer embryos. J Reprod Dev 58:132-139

Kuleshova LL, MacFarlane DR, Trounson AO, Shaw JM (1999) Sugars exert a major influence on the vitrification properties of ethylene glycol-based solutions and have low toxicity to embryos and oocytes. Cryobiology 38:119-130

Lee S, Ryu KJ, Kim B, Kang D, Kim YY, Kim T (2019) Comparison between slow freezing and vitrification for human ovarian tissue cryopreservation and xenotransplantation. Int J Mol Sci 20:3346

Legzdina D, Romanauska A, Nikulshin S, Kozlovska T, Berzins U (2016) Characterization of Senescence of Culture-expanded Human Adipose-derived Mesenchymal Stem Cells. Int J Stem Cells 30:124-136

Len JS, Koh WSD, Tan SX (2019) The roles of reactive oxygen species and antioxidants in cryopreservation. Biosci Rep 39:BSR20191601

Morgunova GV, Kolesnikov AV, Klebanov AA, Khokhlov AN (2016) Senescence-associated $\beta$-galactosidase - a biomarker of aging, DNA damage, or cell proliferation restriction? Gerontology 70: $165-167$

Santos RR, Tharasanit T, Haeften TV, Figueiredo JR, Silva JRV, Hurk RVD (2007) Vitrification of goat preantral follicles enclosed in ovarian tissue by using conventional and solid-surface vitrification methods. Cell Tissue Res 327:167-176

Shivakumar SB, Bharti D, Subbarao RB, Jang SJ, Park JS, Ullah I, Park JK, Byun JH, Park BW, Rho GJ (2016) DMSO- and serum-free cryopreservation of Wharton's jelly tissue isolated from human umbilical cord. J Cell Biochem 117:2397-2412

Son YB, Kang YH, Lee HJ, Jang SJ, Dinesh B, Lee SL, Jeon BG, Park BW, Rho GJ (2021) Evaluation of odonto/osteogenic differentiation potential from different regions derived dental tissue stem cells and effect of $17 \beta$-estradiol on efficiency. BMC Oral Health 21:15

Taylor MJ, Weegman BP, Baicu SC, Giwa SE (2019) New approaches to cryopreservation of cells, tissues, and organs. Transfus Med Hemother 46:197-215

Tinson AH, Singh K, Kuhad KS (2000) Large scale management of camels for embryo transfer. J. Camel Pract Res 7:143-147

Wang Y, Xiao Z, Li L, Fan W, Li SW (2008) Novel needle immersed vitrification: a practical and convenient method with potential advantages in mouse and human ovarian tissue cryopreservation. Hum Reprod 23:2256-2265

Wani NA, Hong S, Vettical BS (2018) Cytoplast source influences development of somatic cell nuclear transfer (SCNT) embryos in vitro but not their development to term after transfer to synchronized recipients in dromedary camels (Camelus dromedarius). Theriogenology 15:137-143

Yang JW, Isla ND, Huselstein C, Kolopp MNS, Li N, Li YP, Ping OYJ, Stoltz JF, Eljaafari A (2006) Evaluation of human MSCs cell cycle, viability and differentiation in micromass culture. Biorheology 43: 489-496

Yeoman RR, Wolf DP, Lee DM (2005) Coculture of monkey ovarian tissue increases survival after vitrification and slow-rate freezing. Fertil Steril 83:1248-1254

Zhang YL, Liu FJ, Zhuang YF, Wang XA, Zhai XW, Li HX, Hong ZY, Chen JJ, Zhong LC, Zhang WC (2012) Blastocysts cloned from the Putian Black pig ear tissues frozen without cryoprotectant at -80 and -196 degrees Celsius for 3 yrs. Theriogenology 78:1166-1170 